Liver, Pancreas and Biliary Tract

\title{
Transjugular Intrahepatic Portosystemic Shunt does not affect the efficacy and safety of direct-acting antivirals in patients with advanced cirrhosis: A real-life, case-control study
}

\author{
Stefano Gitto ${ }^{\mathrm{a}, 1}$, Francesco Vizzutti ${ }^{\mathrm{a}, 1}$, Filippo Schepis ${ }^{\mathrm{b}}$, Laura Turco ${ }^{\mathrm{b}}$, Silvia Aspite ${ }^{\mathrm{a}}$, \\ Giovanni Vitale ${ }^{\mathrm{c}}$, Umberto Arena ${ }^{\mathrm{a}}$, Erica Villa ${ }^{\mathrm{b}}$, Giacomo Laffi ${ }^{\mathrm{a}}$, \\ Wilma Debernardi-Venon ${ }^{d}$, Fabrizio Fanelli ${ }^{\mathrm{e}}$, Pietro Andreone ${ }^{\mathrm{c}}$, \\ Fabio Marra ${ }^{\mathrm{a}, \mathrm{f}, *}$, DAA-TIPS group ${ }^{2}$ \\ a Department of Experimental and Clinical Medicine, University of Florence, Italy \\ b Division of Gastroenterology, Modena Hospital, University of Modena and Reggio Emilia, Modena, Italy \\ ${ }^{c}$ Department of Medical and Surgical Sciences, Center for Study and Research on Chronic Hepatitis, University of Bologna, Italy \\ d Department of Medical Sciences, Division of Gastroenterology and Hepatology, Molinette Hospital, Turin, Italy \\ e Department of Interventional Radiology, Careggi Hospital, Florence, Italy \\ ${ }^{\mathrm{f}}$ Research Center Denothe, University of Florence, Italy
}

\section{A R T I C L E I N F O}

\section{Article history:}

Received 20 August 2018

Accepted 20 November 2018

Available online $\mathrm{xxx}$

\section{Keywords:}

Advanced liver disease

Antiviral therapy

Cirrhosis complications

Portal hypertension

\begin{abstract}
A B S T R A C T
Background: Transjugular Intrahepatic Portosystemic Shunt (TIPS) is a well-established treatment for complications of portal hypertension.

Aims: To analyze the impact of TIPS on virologic response and safety profile in patients treated with direct-acting antivirals (DAAs).

Methods: We analyzed data from HCV-positive cirrhotic patients treated with DAAs. Twenty-one patients with previous TIPS placement were compared with 42 matched subjects without TIPS. Logistic regression was used to identify predictors of hepatic function worsening and adverse events.

Results: No differences were found between the two groups in particular regarding sustained virologic response (92.5 and 97.6\% in TIPS $v s$ no-TIPS, $\mathrm{p}=0.559$ ). Model for End-stage Liver Disease (MELD) of both TIPS and no-TIPS groups declined from baseline to week 24 of follow-up (from $12.5 \pm 3.5$ to $10.8 \pm 3.4$ and from $11.1 \pm 3.5$ to $10.3 \pm 3.4, \mathrm{p}=0.044$ and 0.025$)$. There were no differences in adverse event rates. At univariate analysis, age was associated with MELD increase from baseline to week 24 (OR 1.111, 95\% CI 1.019-1.211, $\mathrm{p}=0.017)$, and patients with higher baseline MELD developed serious adverse events more frequently (OR $0.815,95 \%$ CI $0.658-1.010, \mathrm{p}=0.062$ ). Patients with or without TIPS did not show differences in transplant-free survival.

Conclusion: TIPS placement does not affect virologic response and clinical outcome of patients receiving DAAs.
\end{abstract}

(C) 2018 Editrice Gastroenterologica Italiana S.r.l. Published by Elsevier Ltd. All rights reserved.

\section{Introduction}

About $3 \%$ of the world population is infected with hepatitis $\mathrm{C}$ virus (HCV) infection and 150.000 new infections are registered

\footnotetext{
* Corresponding author at: Department of Experimental and Clinical Medicine, University of Florence, Largo Brambilla, 3, 50134 Florence, Italy.

E-mail address: fabio.marra@unifi.it (F. Marra).

1 These authors contributed equally to the present study.

2 Collaborators of the DAA-TIPS group: Pasquale Apolito ${ }^{a}$, Claudia Campania, Sinan Sadalla ${ }^{a}$, Federica Lombardo ${ }^{a}$, Fabio Contic ${ }^{\mathrm{c}}$, Alessandra Scuteri ${ }^{\mathrm{c}}$.
}

every year in Western countries [1]. Twenty percent of patients with chronic hepatitis C (CHC) develop cirrhosis, and 700.000 deaths associated with complications of liver disease occur every year [2]. Until a few years ago, antiviral therapy based on interferon in association with ribavirin (RBV) was the only approach for infected patients, with modest rates of sustained virologic response (SVR) and significant side effects [3]. During the last few years, new antiviral molecules with a direct action against the virus have been developed, resulting in a dramatic change in the management of $\mathrm{CHC}$ [3]. In fact, most direct-acting antiviral (DAA) schedules induce high SVR rates with excellent safety profiles. At difference with 
interferon-based antiviral therapy, DAAs can be used in patients with advanced liver disease with good SVR rates [4] and a positive impact on patient survival [5]. Nonetheless, Curry et al. [6] reported that in patients in Child-Pugh-Turcotte (CTP) class B treated with DAAs, satisfactory SVR rate (83-94\%) were associated with a relevant number of serious adverse events (SAE) compared to the same regimens in patients with chronic hepatitis or compensated cirrhosis (16-19\% vs 2\%) [6,7].

Transjugular Intrahepatic Portosystemic Shunt (TIPS) is a leading treatment option for the complications of portal hypertension. TIPS is indicated in portal hypertensive bleeding, refractory ascites and vascular liver diseases, resulting in improvement of patient survival [8-12]. Notably, TIPS strongly modifies splanchnic hemodynamics, determining substantial porto-systemic shunting [8]. TIPS placement might influence the pharmacokinetics of different drugs [13-15], and this is theoretically possible also for DAAs. The safety profile represents another open issue, considering the high rates of SAEs in patients with advanced liver disease [6]. These aspects have never been previously investigated.

In this study, we evaluated the impact of TIPS on SVR rate, drug safety profile and liver function in HCV-positive cirrhotic patients treated with DAAs.

\section{Materials and methods}

We analyzed biochemical, virological and clinical data of HCVpositive cirrhotic patients with functioning TIPS, consecutively treated with DAA-based therapies from September 2014 to April 2017. In all cases, TIPS was placed before starting DAA therapy. Cirrhotic patients without TIPS treated with DAAs in the same period of time were used as comparison. They were selected among HCV positive patients consecutively treated in the four tertiary participating centers, according to sex, age ( \pm 3 years) and CTP class (match 1:2). DAA schedule and treatment length were established following the recommendations of the Italian Medicines Agency committee [16].

Enrollment criteria were age $>18$ years and HCV infection as established by both positive serum HCV antibody and serum HCVRNA, using a real-time polymerase chain reaction-based method. Patients had evidence of cirrhosis as assessed by liver biopsy, vibration-controlled transient elastography with FibroScan ${ }^{\circledR}$, or by the presence of complications. Other causes of liver disease including alcohol abuse, and previous liver transplantation were exclusion criteria.

In all patients with previous TIPS placement, shunt patency was documented by radiologic follow-up. All enrolled patients underwent at least one Doppler ultrasound within six months from the beginning of antiviral therapy with the following signs of optimal function [17]: whole color saturation in three segments of the stent (portal, midportion, and hepatic sites) without any evidence of filling defects or focal aliasing; high-velocity turbulent flow into the stent with a gradual velocity shift during respiration; hepatofugal flow in the portal vein branches; hepatic artery flow increase.

Laboratory assessment, including HCV-RNA levels, was conducted at baseline, end of antiviral treatment, and 12 and 24 weeks after the end of DAA. Model for End-Stage Liver Disease (MELD) and MELD corrected by serum sodium (MELD-Na) were calculated at all time points. Delta values were calculated (week 24 of followup - baseline) for all the main laboratory values. HCV-RNA levels were measured with COBAS $^{\circledR}$ AmpliPrep/COBAS ${ }^{\circledR}$ TaqMan $^{\circledR}$ HCV Quantitative Test, v2.0 (limit $15 \mathrm{IU} / \mathrm{ml}$ ).

We registered DAA-related and unrelated adverse events (AEs) (untoward medical occurrence associated with the use of antiviral therapy) and SAEs (death, a life-threatening AE, inpatient hospitalization or prolongation of existing hospitalization, a persistent or significant incapacity or substantial disruption of the ability to conduct normal life functions) [18]. The study protocol conformed to the ethical guidelines of the 1975 Declaration of Helsinki. Patients gave their written informed consent to undergo antiviral therapy with DAAs.

\subsection{Statistical analysis}

A physician trained in statistics encoded all samples into a dedicated database in an anonymous form. Data are expressed as the mean ( \pm standard deviation) or median (with range), as applicable. Confidence interval $(\mathrm{CI})$ is shown where appropriate. Comparisons between groups were made by Chi square-test and Fisher's exact test for qualitative variables, and Mann-Whitney U-test for continuous ordinal data, respectively. Wilcoxon test was utilized to assess tendencies and specific rank test for significance. Logistic regression was performed to identify the predictors of liver function impairment, AEs and SAEs. Presence of TIPS was analyzed as potential predictor in all outcome analyses. A significance level of 0.05 was considered for all tests. The SPSS software version 21.0 (MJ Norusis, Chicago, US) was used for all statistical analyses.

\section{Results}

\subsection{Baseline characteristics}

We enrolled $21 \mathrm{HCV}$-positive cirrhotic patients with previous TIPS placement and 42 cirrhotic subjects without TIPS, matched for gender, age and CTP class. The main demographic and baseline clinical characteristics of the two groups (TIPS and no-TIPS) are summarized in Table 1 . Additional clinical and technical data of patients included in the TIPS group are reported in Supplementary Table 1 . Notably, a fraction of patients had under-dilated TIPS according to an approach described in a recently published study [19]. Patients with TIPS and the counterparts were comparable for all other clinical and virologic characteristics. Genotype $1 \mathrm{~b}$ was the most represented, and sofosbuvir/ledipasvir the most frequently used antiviral schedule. RBV was added to DAA in approximately half of the cases, in both groups.

\subsection{Virologic response}

All patients concluded the antiviral therapy. All patients, regardless of the presence of TIPS, reached the SVR12 but not all maintained the virologic response at 24 weeks of follow-up (SVR24 92.5\% vs $97.6 \%$ in TIPS and no-TIPS, respectively, $\mathrm{p}=0.559$ ). One patient in each group had a virologic relapse: in the TIPS group, a 49 years old male, genotype 4 , CTP class A, treated for 24 weeks with sofosbuvir/ledipasvir without RBV; in the no-TIPS group, a 42 years old male, genotype $1 \mathrm{a}$, CTP class B, treated for 24 weeks with sofosbuvir/ledipasvir without RBV.

\subsection{Changes in laboratory parameters and liver function}

The behavior of laboratory tests at baseline (T0), end of treatment (EOT) and week 24 of follow-up (W24) is reported in Table 2. Patients with TIPS and no-TIPS were in general comparable for all laboratory assessments, although those with TIPS had higher median values of total bilirubin at EOT and at W24 in comparison with patients without TIPS.

Analyzing the modifications along the three time points, aminotransferases and $\gamma$ GT levels decreased in both groups, while total bilirubin significantly declined only in patients without TIPS. Mean MELD scores at each time point and their trend during and after antiviral therapy are reported in Table 2 and in Fig. 1. Patients of the two groups had statistically equivalent MELD and MELD-Na 
Table 1

Baseline characteristics of patients included in the TIPS and no-TIPS groups.

\begin{tabular}{|c|c|c|c|}
\hline & TIPS & no-TIPS & $\begin{array}{l}\chi 2 \text {-Fisher/MW-U } \\
\text { P value }\end{array}$ \\
\hline $\mathrm{N}$ & 21 & 42 & \\
\hline Male $\mathrm{n}(\%)$ & $18(85.7)$ & $36(85.7)$ & 1.000 \\
\hline Age (years, mean $\pm S D$ ) & $58 \pm 10$ & $61 \pm 10$ & 0.385 \\
\hline \multicolumn{4}{|l|}{ Genotype n (\%) } \\
\hline $1 \mathrm{a}$ & $4(19)$ & $8(19)$ & 1.000 \\
\hline $1 \mathrm{~b}$ & $9(42.9)$ & $21(50)$ & 0.395 \\
\hline 2 & $1(4.8)$ & $2(4.8)$ & 1.000 \\
\hline 3 & $3(14.3)$ & $8(19)$ & 0.564 \\
\hline 4 & $4(19)$ & $3(7.1)$ & 0.160 \\
\hline HCV-RNA (UI/ml) (median, R) & $352977.8740-12723486$ & 380000. 2750-12295107 & 0.683 \\
\hline \multicolumn{4}{|l|}{ CTP score $\mathrm{n}(\%)$} \\
\hline A6 & $9(42.8)$ & $18(42.8)$ & 1.000 \\
\hline B7 & $3(14.3)$ & $15(35.7)$ & 0.137 \\
\hline B8 & $8(38.1)$ & $5(11.9)$ & 0.023 \\
\hline B9 & $0(0)$ & $2(4.8)$ & 0.548 \\
\hline $\mathrm{C} 10$ & $1(4.8)$ & $2(4.8)$ & 1.000 \\
\hline $\operatorname{MELD}($ mean \pm SD $)$ & $12.5 \pm 3.5$ & $11.1 \pm 3.5$ & 0.177 \\
\hline MELD-Na $($ mean \pm SD) & $13.6 \pm 3.5$ & $12.4 \pm 5.2$ & 0.055 \\
\hline \multicolumn{4}{|l|}{ DAA regimen $\mathrm{n}(\%)$} \\
\hline SOF/SIM & 0 & $2(4.8)$ & 0.441 \\
\hline SOF/LPV & $11(52.4)$ & $18(42.9)$ & 0.327 \\
\hline SOF/DCV & $7(33.3)$ & $14(33.3)$ & 1.000 \\
\hline SOF & $2(9.5)$ & $3(7.1)$ & 0.545 \\
\hline OBV/PTV/RTV/DSV & 0 & $4(9.5)$ & 0.188 \\
\hline OBV/PTV/RTV & $1(4.8)$ & $1(2.4)$ & 0.559 \\
\hline RBV n (\%) & $9(42.9)$ & $20(47.6)$ & 0.465 \\
\hline Length of therapy $12 / 24$ week n (\%) & $0(0) / 21(100 \%)$ & $6(14.3) / 36(85.7)$ & 0.077 \\
\hline
\end{tabular}

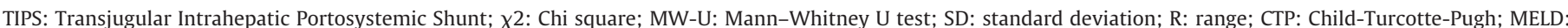

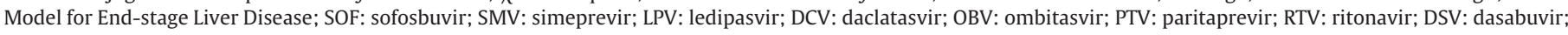
RBV: ribavirin.

Table 2

Laboratory parameters in cirrhotic patients with or without TIPS undergoing therapy with DAA.

\begin{tabular}{|c|c|c|c|c|c|c|}
\hline & \multicolumn{3}{|l|}{ TIPS } & \multicolumn{3}{|l|}{ no-TIPS } \\
\hline & T0 & EOT & W24 & T0 & EOT & W24 \\
\hline $\operatorname{PLT}\left(\times 10^{9} / \mathrm{mmc}\right)($ median, $\mathrm{R})$ & 79. 36-161 & 78. 33-172 & 78. 36-164 & 98. 19-222 & $92,19-259$ & 98. 20-206 \\
\hline Bilirubin (mg/dl) (median, R) & 1.8. $0.8-7.2$ & 1.7. $1.1-4.6^{\mathrm{b}}$ & 1.3. $0.5-3.4^{\mathrm{b}}$ & 1.3. $0.4-8.6$ & $1.3,0.2-6.7^{\mathrm{a}}$ & 1. $0.4-5.5^{\mathrm{a}}$ \\
\hline AST (IU/ml) (median, R) & $61.28-154$ & $31.14-63^{\mathrm{a}}$ & $30.17-55^{a}$ & 73. 21-519 & $28,16-66^{\mathrm{a}}$ & $25.13-71^{\mathrm{a}}$ \\
\hline ALT (IU/ml) (median, R) & 47. 21-151 & 23. $9-48^{\mathrm{a}}$ & 21. $10-52^{\mathrm{a}}$ & $60.11-405$ & $20,11-48^{a}$ & 21. $10-58^{\mathrm{a}}$ \\
\hline$\gamma \mathrm{GT}(\mathrm{IU} / \mathrm{ml})($ median, $\mathrm{R})$ & 53. 10-303 & 36. $7-246^{a}$ & $33.8-120^{\mathrm{a}}$ & $67.16-303$ & $41,12-121^{\mathrm{a}}$ & $42.12-119^{a}$ \\
\hline ALP (IU/ml, median, R) & 100. $62-233$ & 99. 46-193 & $104.57-200^{\mathrm{b}}$ & 126. 58.490 & $123,59-635^{a}$ & 157. $22-801$ \\
\hline Creatinine $(\mathrm{mg} / \mathrm{dl}$, mean $\pm \mathrm{SD})$ & $0.9 \pm 0.2$ & $0.8 \pm 0.3$ & $1 \pm 0.4$ & $0.9 \pm 0.3$ & $0.9 \pm 0.3$ & $0.9 \pm 0.3$ \\
\hline MELD score $($ mean $\pm S D)$ & $12.5 \pm 3.5$ & $12.7 \pm 3^{b}$ & $10.8 \pm 3.4^{\mathrm{a}}$ & $11.1 \pm 3.5$ & $10.5 \pm 3.4^{\mathrm{a}}$ & $10.3 \pm 3.4^{a}$ \\
\hline MELD-Na score (mean \pm SD) & $13.6 \pm 3.5$ & $13.7 \pm 3.2^{\mathrm{b}}$ & $11.7 \pm 3.4^{\mathrm{a}}$ & $12.4 \pm 5.2$ & $11 \pm 4.2$ & $10.4 \pm 3.4^{\mathrm{a}}$ \\
\hline
\end{tabular}

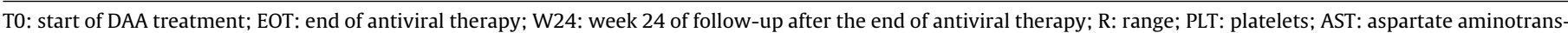
ferase; ALT: alanine aminotransferase; $\gamma \mathrm{GT}$ : gamma glutamyl transferase; ALP: alkaline phosphatase; MELD: Model for End-Stage Liver Disease.

a Significant difference with respect to T0 (Wilcoxon test, $\mathrm{p}<0.05$ ).

b Significant difference compared to the same time point in no-TIPS (Mann-Whitney U test, $\mathrm{p}<0.05$ ).

Table 3

Comparison of changes in laboratory parameters before and after antiviral treatment in cirrhotic patients with or without TIPS

\begin{tabular}{|c|c|c|c|}
\hline Delta value (W24 - T0) & TIPS & no-TIPS & P value \\
\hline$\Delta$ PLT $\left(\times 10^{9} / \mathrm{mmc}\right)$ median $(\mathrm{R})$ & $+2(-61$ to +54$)$ & $+4(-42$ to +49$)$ & 0.406 \\
\hline$\Delta$ Bilirubin $(\mathrm{mg} / \mathrm{dl})$ median $(\mathrm{R})$ & $-0.4(-6.7$ to +2.5$)$ & $-0.3(-15$ to +1$)$ & 0.994 \\
\hline$\Delta \mathrm{AST}(\mathrm{IU} / \mathrm{ml})$ median $(\mathrm{R})$ & $-29(-137$ to +9$)$ & $-48(-448$ to -1$)$ & 0.055 \\
\hline$\Delta$ ALT $(\mathrm{IU} / \mathrm{ml})$ median $(\mathrm{R})$ & $-25(-144$ to +12$)$ & $-41(-347$ to +35$)$ & 0.079 \\
\hline$\Delta \gamma \mathrm{GT}(\mathrm{IU} / \mathrm{ml})$ median $(\mathrm{R})$ & $-20(-141$ to +37$)$ & $-22(-218$ to +99$)$ & 0.569 \\
\hline$\triangle \mathrm{ALP}(\mathrm{IU} / \mathrm{ml})$ median $(\mathrm{R})$ & $-6(-141$ to +39$)$ & $+8(-153$ to +347$)$ & 0.354 \\
\hline$\Delta$ Creatinine $(\mathrm{mg} / \mathrm{dl})$ mean $\pm S D$ & $+0.1 \pm 0.2$ & $+0.05 \pm 0.2$ & 0.331 \\
\hline$\Delta \mathrm{MELD}$ score mean $\pm \mathrm{SD}$ & $-1.7 \pm 3.4$ & $-0.7 \pm 2.6$ & 0.039 \\
\hline$\Delta$ MELD-Na score mean $\pm S D$ & $-2 \pm 3.9$ & $-2 \pm 5.1$ & 0.433 \\
\hline
\end{tabular}

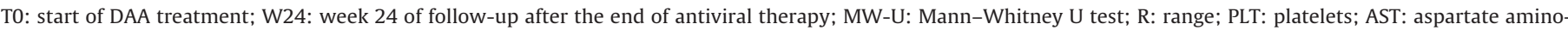

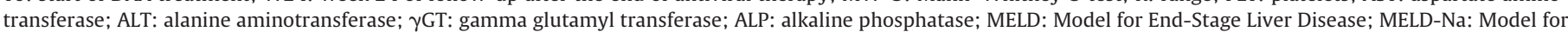
End-Stage Liver Disease corrected by serum sodium.

scores at T0 and W24. Patients with TIPS showed a significantly higher mean MELD and MELD-Na at EOT in comparison with subjects with no-TIPS. Mean MELD score dropped from T0 to EOT and from T0 to W24 in the control group. Patients with TIPS showed a significant decrease of mean MELD from T0 to W24 but not from T0 to EOT. In both groups, MELD-Na significantly decreased from 


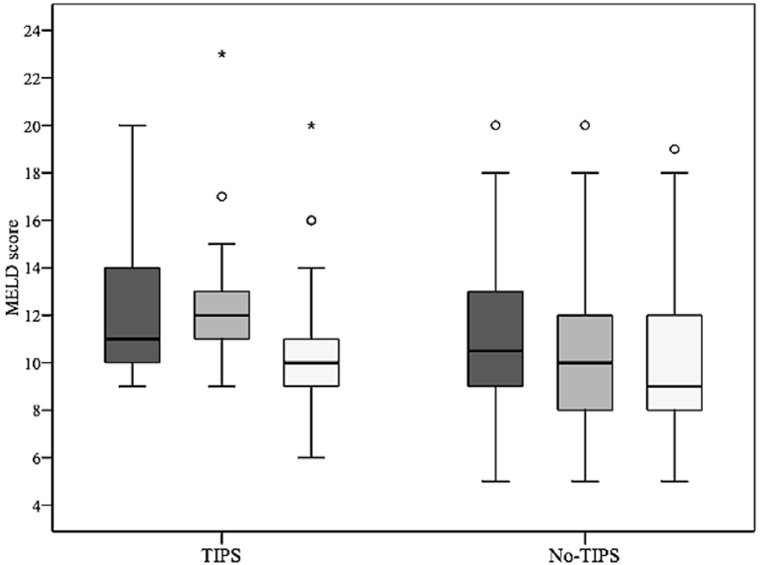

Fig. 1. Distribution of MELD score (ranging from 6 to 40, with higher values indicating more advanced liver disease) according to the previous TIPS placement. MELD at beginning of antiviral treatment (dark grey bar), MELD calculated at the end of antiviral therapy (light grey bar), MELD at week 24 from the end of treatment (white bar). The horizontal bar inside the box indicates the median value. The points represent the outliers, defined as values not falling in the inner fences. The asterisks are the extreme outliers, defined as values more than three times the height of the boxes.

T0 to W24. Delta values (W24 - T0) of the analyzed laboratory parameters are reported in Table 3. Patients with TIPS showed a significantly greater mean delta-MELD than patients without TIPS.

MELD score declined from T0 to W24 in the vast majority of patients while it increased in $14.3 \%$ of patients with TIPS (3/21) and in $16.7 \%$ of no-TIPS $(7 / 42)(\mathrm{p}=0.559)$. At univariate analysis, only patients' age was significantly associated with the increase in MELD score (from T0 to W24) [Odds Ratio (OR) 1.111, 95\% CI 1.019-1.211, $\mathrm{p}=0.017]$. In particular, mean age was $67 \pm 7$ in patients with increased MELD, and $59 \pm 10$ years in those who decreased MELD. The presence of TIPS or time between TIPS placement and start of DAA therapy did not emerge as significant variables either in the preliminary univariate test or in the multivariate model.

\subsection{Safety}

The main safety outcomes are summarized in Table 4. Comparing TIPS and no-TIPS, no significant differences were found in the incidence of $\mathrm{AE}$. The most common $\mathrm{DAA}$-related $\mathrm{AE}$ was fatigue. HCC occurrence during antiviral therapy was the most frequent SAE unrelated to DAA. No DAA-related SAEs were registered. No patients discontinued treatment prematurely because of an $A E$ or SAE.

Male gender was the only feature that tended to be associated with the occurrence of $\mathrm{AE}$ (OR $0.148,95 \% \mathrm{CI} 0.018-1.241, \mathrm{p}=0.078$ ). AEs were reported in $40.4 \%$ of males $(21 / 52)$ and in $9.1 \%$ of females (1/11). No predictors of DAA-related AE were detected.

At univariate analysis for the predictors of SAE, MELD score at T0 emerged as the only factor with a P value $<0.1$ (OR $0.815,95 \% \mathrm{CI}$ $0.658-1.010, p=0.062$ ). Patients who experienced a SAE showed a mean baseline MELD score of $14 \pm 5$, compared to $11 \pm 3$ in the other patients. The presence of TIPS or time between TIPS placement and start of DAA therapy did not emerge as significant variables either in the preliminary univariate test or in the multivariate model.

\subsection{Long-term outcome}

No patient died or was transplanted during the antiviral therapy. During the 2-year follow-up period, 1 patient died (no-TIPS group) while 3 patients with TIPS and 1 no-TIPS were transplanted. Two-year cumulative survival of patients with TIPS was $100 \%$ while
Table 4

Adverse events in cirrhotic patients with or without TIPS undergoing treatment with DAA.

\begin{tabular}{|c|c|c|c|}
\hline & TIPS & no-TIPS & $\begin{array}{l}\chi 2 \text {-Fisher } \\
\text { P value }\end{array}$ \\
\hline $\mathrm{N}$ & 21 & 42 & \\
\hline AEs (overall) n (\%) & $9(42.9)$ & $13(31)$ & 0.255 \\
\hline AEs (DAA-related) n (\%) & $3(14.3)$ & $10(23.8)$ & 0.297 \\
\hline Fatigue & $3(100)$ & $7(70)$ & \\
\hline Skin rash & 0 & $2(20)$ & \\
\hline Nausea & 0 & $1(10)$ & \\
\hline AEs (not DAA-related) n (\%) & $6(28.6)$ & $3(7.1)$ & 0.031 \\
\hline Ascites & $2(33.3)$ & 0 & \\
\hline Rectal bleeding & $1(16.7)$ & 0 & \\
\hline Skin infection & $1(16.7)$ & 0 & \\
\hline Hepatic Hencephalopathy & $1(16.7)$ & 0 & \\
\hline Depression & $1(16.7)$ & $2(66.7)$ & \\
\hline Anaemia & 0 & $1(33.3)$ & \\
\hline Withdrawal of antiviral for $\mathrm{AE}$ & 0 & 0 & 1.000 \\
\hline SAEs (overall) n (\%) & $1(4.8)$ & $6(14.3)$ & 0.248 \\
\hline SAEs (DAA-related) n (\%) & 0 & 0 & 1.000 \\
\hline SAEs (not DAA-related) n (\%) & $1(4.8)$ & $6(14.3)$ & 0.248 \\
\hline $\mathrm{HCC}$ & $1(100)$ & $3(50)$ & \\
\hline Liver failure & 0 & $1(16.7)$ & \\
\hline SBP & 0 & $1(16.7)$ & \\
\hline Portal thrombosis & 0 & $1(16.7)$ & \\
\hline Withdrawal of antiviral for SAE n (\%) & 0 & 0 & 1.000 \\
\hline Death during treatment n (\%) & 0 & 0 & 1.000 \\
\hline Death during 2-year follow-up n (\%) & 0 & $1(2.4)$ & 0.667 \\
\hline LT during treatment $\mathrm{n}(\%)$ & 0 & 0 & 1.000 \\
\hline LT during 2-year follow-up n (\%) & $3(14.3)$ & $1(2.4)$ & 0.104 \\
\hline
\end{tabular}

$\chi 2$ : Chi square; AE: adverse event; DAA: direct acting antivirals; SAE. serious adverse event; HCC: hepatocellular carcinoma; SBP: spontaneous bacterial peritonitis; LT: Liver Transplant.

survival of no-TIPS was $97.4 \%(\mathrm{p}=0.549)$. Death and transplantation occurred in all cases for progression of the underlying liver disease, and were not related to DAA therapy. The patient who died was a 60 years old male, genotype $1 \mathrm{~b}$, CTP B, treated with ombitasvir/paritaprevir/ritonavir plus dasabuvir and RBV for 12 weeks with achievement of RVR, SVR12 and SVR24. The cause of death was a complication of bowel obstruction.

\section{Discussion}

Patients with HCV-related advanced liver disease might benefit from both TIPS placement and DAA therapy [6,8-12]. However, TIPS leads to porto-systemic shunting [8] and its impact on drug pharmacokinetics and consequently on efficacy and safety is uncertain. While DAA therapy is generally safe in CHC and compensated cirrhosis [6,7], in patients with advanced liver disease, relevant rates of SAE were observed [6,7]. In this study, we demonstrated for the first time that patients with previous placement of TIPS can achieve optimal rates of SVR24 (92.5\%) and a significant decrease in MELD and MELD-Na score from T0 to W24. In particular, both SVR rate and the improvement of liver function in patients with prior placement of TIPS treated with DAA are coherent with previous studies in patients with advanced cirrhosis without TIPS $[6,20]$. It should be noted that in patients with TIPS the decline of MELD was somehow slower than in patients without TIPS. However, in patients with TIPS a significantly higher delta-MELD than in patients without TIPS was observed.

Notably, at two years of follow-up, patients with and without TIPS showed a comparable percentage of hard clinical outcomes (LT and death). Surprisingly, patients with TIPS tended to develop a lower number of DAA-related AEs and SAEs with respect to patients without TIPS, although this difference was not significant. While it is conceivable that the reduction in portal pressure could have an impact on some of the AEs, data from larger series of patients are necessary before definitive conclusions on this issue may be drawn. 
We also analyzed the whole population of patients with severe liver disease, irrespective of TIPS, trying to identify independent predictors of MELD increase from T0 to W24, and of AEs and SAEs. Indeed, while in the majority of patients MELD score decreased from T0 to the end of follow-up (W24), in a subgroup of patients the score increased (14.3\% of patients with TIPS and $16.7 \%$ without TIPS). Elderly patients were at higher risk of MELD increase, confirming that age is a determinant of the likelihood to improve liver function, possible due to differences in hepatic functional reserve. These results are in agreement with those of Conti et al. [21], who demonstrated that patients with advanced fibrosis or cirrhosis aged $\geq 65$ years, and treated with DAA, had a worsening of CTP class, at difference from younger patients. Gender emerged as a borderline predictor of the appearance of AE during DAA treatment. In fact, males showed four times as many AEs as females. Although this is an original and potentially interesting finding, the low number of females enrolled in this study does not allow to draw any definitive conclusions. Further studies should explore possible gender differences in terms of SVR and safety profile in this population.

Patients with higher MELD score at T0 displayed a higher risk of SAE. This finding is not surprising since only SAEs associated with advanced liver disease itself and not related to DAAs were registered. Notably, we could not analyze the possible predictors of SVR or of transplant or death due to the low number of events.

We have to acknowledge a few limitations of this study, including the relatively small number of patients enrolled. However, this is the first real-life study analyzing whole laboratory, virological, and clinical outcomes of patients treated with DAA after TIPS. Because DAAs have been introduced in clinical practice only few years ago, the length of follow-up reported in the present study (2 years) is adequate to confirm the excellent results of DAA-based therapy also in patients with advanced liver disease. The observational design of the study represents a further limitation, although the 1:2 matching can assure an acceptable strength of the study design. The study adds real life-based knowledge in the context of advanced cirrhosis, a condition where additional post-registration information may still be of value [22]. Future studies should be undertaken to evaluate whether the outcomes observed in TIPS patients treated with DAA are different from those of patients with TIPS not undergoing antiviral therapy. In the cohorts available in our centers, these latter patients had TIPS placed several years before and this would create a bias in the comparison. Another limitation of this study is the lack of pharmacokinetic data in the two groups of patients. These data, which could establish whether, and to what extent, DAAs plasma levels are different in patients with TIPS, deserve to be obtained in future studies.

In conclusion, DAA-based antiviral therapy may be safely and effectively used after TIPS placement. Cirrhotic patients with and without TIPS treated with DAA have similar long-term outcomes and comparable chances to improve their liver function.

\section{Conflict of interest}

FV has received undisclosed research grant and lecture fees from Gore.

FS has received undisclosed research grant and support grant from Gore.

EV has been consultant and speaker for MSD, AbbVie, Gilead Science, BMS.

PA has been in advisory board for MSD, Gilead Sciences, AbbVie, BMS, Intercept and has received research grant form MSD, AbbVie, Gilead Sciences, BMS.

FM has been consultant for AbbVie, Gilead Science, Menarini, ViiV, Novo Nordisk, Astrazeneca, Bayer, Allergan, Intercept and has received support grant from Alpha Sigma, Bayer, AbbVie, Kedrion.

\section{Appendix A. Supplementary data}

Supplementary data associated with this article can be found, in the online version, at https://doi.org/10.1016/j.dld.2018.11.015.

\section{References}

[1] Global Burden of Hepatitis CWG. Global burden of disease (GBD) for hepatitis C. J Clin Pharmacol 2004;44:20-9.

[2] Guidelines for the screening, care and treatment of persons with hepatitis $C$ infection. http://www.who.com. [Accessed 3 October 2016].

[3] European Association for the Study of the Liver. EASL recommendations on treatment of hepatitis C 2018. J Hepatol 2018;69:461-511, http://dx.doi.org/ 10.1016/j.jhep.2018.03.026, pii: S0168-8278(18)31968-8.

[4] Mangia A, Losappio R, Cenderello G, Potenza D, Mazzola M, De Stefano G, et al. Real life rates of sustained virological response (SVR) and predictors of relapse following DAA treatment in genotype 3 (GT3) patients with advanced fibrosis/cirrhosis. PLoS One 2018;13:e0200568, http://dx.doi.org/10.1371/journal. pone.0200568.

[5] Backus LI, Belperio PS, Shahoumian TA, Mole LA. Impact of sustained virologic response with direct-acting antiviral treatment on mortality in patients with advanced liver disease. Hepatology 2019;69:487-97, http://dx.doi.org/10. 1002/hep. 29408.

[6] Curry MP, O’Leary JG, Bzowej N, Muir AJ, Korenblat KM, Fenkel JM, et al. Sofosbuvir and velpatasvir for HCV in patients with decompensated cirrhosis. N Engl J Med 2015;373:2618-28, http://dx.doi.org/10.1056/NEJMoa1512614.

[7] Feld JJ, Jacobson IM, Hézode C, Asselah T, Ruane PJ, Gruener N, et al. Sofosbuvir and velpatasvir for HCV genotype 1, 2, 4, 5, and 6 infection. N Engl J Med 2015;373:2599-607, http://dx.doi.org/10.1056/NEJMoa1512610.

[8] Boyer TD, Haskal ZJ. The role of transjugular intrahepatic portosystemic shunt in the management of portal hypertension. Hepatology 2005;41:386-400.

[9] Fagiuoli S, Bruno R, Debernardi Venon W, Schepis F, Vizzutti F, Toniutto P, et al. AISF TIPS special conference. Consensus conference on TIPS management: techniques, indications, contraindications. Dig Liver Dis 2017;49:121-37.

[10] Salerno F, Cammà C, Enea M, Rössle M, Wong F. Transjugular intrahepatic portosystemic shunt for refractory ascites: a meta-analysis of individual patient data. Gastroenterology 2007;133:825-34.

[11] García-Pagán JC, Caca K, Bureau C, Laleman W, Appenrodt B, Luca A, et al. Early use of TIPS in patients with cirrhosis and variceal bleeding. N Engl J Med 2010;24:2370-9.

[12] Bureau C, Thabut D, Oberti F, Dharancy S, Carbonell N, Bouvier A, et al. Transjugular intrahepatic portosystemic shunts with covered stents increase transplant-free survival of patients with cirrhosis and recurrent ascites. Gastroenterology 2017;152:157-63.

[13] Chalasani N, Gorski JC, Patel NH, Hall SD, Galinsky RE, et al. Hepatic and intestinal cytochrome P450 3A activity in cirrhosis: effects of transjugular intrahepatic portosystemic shunts. Hepatology 2001;34:1103-8.

[14] Spriet I, Meyfroidt G, Maleux G, Verslype C, Willems L. The impact of a transjugular intrahepatic portosystemic shunt on the pharmacokinetics of caspofungin in a critically ill patient. Pharmacology 2012;90:247-50, http:// dx.doi.org/10.1159/000342906.

[15] De Winter S, Verelst S, Wauters J, Van der Linden L, Verslype C, Willems L, et al. Pharmacokinetic changes after placement of a transjugular intrahepatic portosystemic shunt. Eur J Clin Pharmacol 2014;70:377-8, http://dx.doi.org/ 10.1007/s00228-013-1629-y.

[16] http://www.agenziafarmaco.gov.it/.

[17] Ricci P, Cantisani V, Lombardi V, Alfano G, D’Ambrosio U, Menichini G, et al. Is color-Doppler US a reliable method in the follow-up of transjugular intrahepaticportosystemic shunt (TIPS)? J Ultrasound 2007;10:22-7, http://dx.doi.org/ 10.1016/j.jus.2007.02.005.

[18] https://www.accessdata.fda.gov/scripts/cdrh/cfdocs/cfcfr/cfrsearch. cfm?fr=312.32. [Accessed 1 April 2017].

[19] Schepis F, Vizzutti F, Garcia-Tsao G, Marzocchi G, Rega L, De Maria N, et al. Under-dilated TIPS associate with efficacy and reduced encephalopathy in a prospective, non-randomized study of patients with cirrhosis. Clin Gastroenterol Hepatol 2018;16, http://dx.doi.org/10.1016/j.cgh.2018.01.029, 1153-62.e7.

[20] Ji F, Wang W, Dang S, Wang S, Li B, Bai D, et al. Outcomes after sofosbuvircontaining regimens for hepatitis $C$ virus in patients with decompensated cirrhosis: a real-world study. Infect Agents Cancer 2017:48, http://dx.doi.org/ 10.1186/s13027-017-0158-1.

[21] Conti F, Brillanti S, Buonfiglioli F, Vukotic R, Morelli MC, Lalanne C, et al. Safety and efficacy of direct-acting antivirals for the treatment of chronic hepatitis $\mathrm{C}$ in a real-world population aged 65 years and older. J Viral Hepat 2017;24:454-63.

[22] Barone M, Iannone A, Shahini E, Ippolito AM, Brancaccio G, Morisco F, et al. A different perspective on sofosbuvir-ledipasvir treatment of patients with HCV genotype 1b cirrhosis: the ital-c network study. J Viral Hepat 2018;25:56-62. 